\title{
The use of cryo electron microscopy to reveal the distribution and 3D structure of AIDS virus envelope spikes P Zhu' ${ }^{1}$, J Liu ${ }^{1}$, J Bess Jr², E Chertova², JD Lifson² ${ }^{2}$ H Grisé ${ }^{1}$, G Ofek ${ }^{3}$, KA Taylor ${ }^{1}$ and KH Roux*1
}

\author{
Address: ${ }^{1}$ Florida State University, Tallahassee Florida, USA, ${ }^{2}$ SAIC Frederick, Inc., National Cancer Institute at Frederick, Frederick, Maryland, USA \\ and ${ }^{3}$ National Institute of Allergy and Infectious Diseases, National Institutes of Health, Bethesda, Maryland, USA \\ * Corresponding author
}

from 2006 International Meeting of The Institute of Human Virology

Baltimore, USA. 17-2I November, 2006

Published: 2I December 2006

Retrovirology 2006, 3(SuppI I):S5 doi:10.1 186/1742-4690-3-SI-S5

(c) 2006 Zhu et al; licensee BioMed Central Ltd.

Though crystal structures for Env subunit fragments have been described, the overall structure of the Env spike and its distribution on virions has remained obscure. To address this issue, we have used cryoelectron microscopy (cryoEM) tomography to image unfixed and unstained HIV-1 and SIV virions in vitreous ice.

Mutant (truncated TM tail) SIV virions show a surface fairly well saturated with $73 \pm 25$ rather uniformly distributed Env spikes, in line with our previous data. In striking contrast to SIV, yet consistent with our earlier results, cryoEM analysis of HIV-1 (MN) virions, which have a native non-mutated full-length tail, shows only $14 \pm 7$ Env/particle. An analysis of the Env spike distribution on HIV-1 virions shows a significantly higher proportion of spikes within clusters than predicted by chance alone. The clustering of Env spikes has implications for viral fusion and antibody-mediated neutralization mechanisms.

To further investigate the surface features of these virions, we have produced a $3.2 \mathrm{~nm}$ resolution model of the SIV spike based on cryoEM tomogram 3D volume averages of over 6,000 spikes. The gp120 "head" of each subunit of the trimeric SIV spike contains a primary mass, with two secondary lobes. Our model shows that the TM "stalk" of each trimer spike is composed of three independent legs that project obliquely from the trimer head, tripod like. The model was fitted with available atomic structures of the unliganded gp120 core and the HIV-1 gp41 2F5 and
4E10 peptide epitopes and associated Fab fragments. The unusual open leg configuration of TM has implications for the design of future soluble Env trimer vaccine candidates. It also suggests a mechanistic explanation for the events leading up to membrane fusion wherein the three unassociated membrane-spanning domains (MSDs) and membrane-associated elements of the membrane proximal external domains (MPEDs) of a single spike can, collectively, encompass and disrupt the integrity of a relatively large area of the membrane, possibly by pulling upon and drawing the outer leaflet toward the target cell membrane upon triggering. 